BMJ Open

Diabetes

Research

\& Care

\title{
Intraperitoneal, subcutaneous and intravenous glucagon delivery and subsequent glucose response in rats: a randomized controlled crossover trial
}

\author{
Ilze Dirnena-Fusini, ${ }^{1}$ Marte Kierulf Åm, ${ }^{1,2}$ Anders Lyngvi Fougner, ${ }^{3}$ \\ Sven Magnus Carlsen, ${ }^{1,2}$ Sverre Christian Christiansen ${ }^{1,2}$
}

\begin{abstract}
To cite: Dirnena-Fusini I, Åm MK, Fougner AL, et al. Intraperitoneal, subcutaneous and intravenous glucagon delivery and subsequent glucose response in rats: a randomized controlled crossover trial. BMJ Open Diab Res Care 2018;6:e000560. doi:10.1136/ bmjdrc-2018-000560
\end{abstract}

\section{- Additional material is} published online only. To view please visit the journal online (http://dx.doi.org/10.1136/ bmjdrc-2018-000560).

ID-F and MKÅm are joint first authors.

Data from this study were presented as an abstract and poster at the 11th International Conference on 'Advanced Technology and Treatment for Diabetes', February 14-17, 2018, Vienna, Austria.

Received 28 May 2018 Revised 31 August 2018 Accepted 6 October 2018

Check for updates

C Author(s) (or their employer(s)) 2018. Re-use permitted under CC BY-NC. No commercial re-use. See rights and permissions. Published by BMJ.

For numbered affiliations see end of article.

Correspondence to Ilze Dirnena-Fusini; ilze.dirnena-fusini@ntnu.no

\section{ABSTRACT}

Objective Hypoglycemia is a frequent and potentially dangerous event among patients with diabetes mellitus type 1. Subcutaneous glucagon is an emergency treatment to counteract severe hypoglycemia. The effect of intraperitoneal glucagon delivery is sparsely studied. We performed a direct comparison of the blood glucose response following intraperitoneally, subcutaneously and intravenously administered glucagon.

Research design and methods This is a prospective, randomized, controlled, open-label, crossover trial in 20 octreotide-treated rats. Three interventions, 1 week apart, in a randomized order, were done in each rat. All 20 rats were given intraperitoneal and subcutaneous glucagon injections, from which 5 rats were given intravenous glucagon injections and 15 rats received placebo (intraperitoneal isotonic saline) injection. The dose of glucagon was $5 \mu \mathrm{g} / \mathrm{kg}$ body weight for all routes of administration. Blood glucose levels were measured before and until $60 \mathrm{~min}$ after the glucagon/placebo injections. Results Compared with placebo-treated rats, a significant increase in blood glucose was observed 4 min after intraperitoneal glucagon administration ( $p=0.009)$, whereas after subcutaneous and intravenous glucagon administration significant increases were seen after $8 \mathrm{~min}$ $(p=0.002$ and $p<0.001$, respectively). In intraperitoneally treated compared with subcutaneously treated rats, the increase in blood glucose was higher after $4 \mathrm{~min}(\mathrm{p}=0.019)$ and lower after $40 \mathrm{~min}(\mathrm{p}=0.005)$ and $50 \mathrm{~min}(\mathrm{p}=0.011)$. The maximum glucose response occurred earlier after intraperitoneal compared with subcutaneous glucagon injection ( $25 \mathrm{~min}$ vs $35 \mathrm{~min} ; \mathrm{p}=0.003$ ).

Conclusions Glucagon administered intraperitoneally gives a faster glucose response compared with subcutaneously administered glucagon in rats. If repeatable in humans, the more rapid glucose response may be of importance in a dual-hormone artificial pancreas using the intraperitoneal route for administration of insulin and glucagon.

\section{INTRODUCTION}

Patients with diabetes mellitus type 1 (DM1) are treated with either repeated or continuous subcutaneous delivery of insulin to counteract hyperglycemia. Improved glucose control

\section{Significance of this study}

What is already known about this subject?

- Glucagon is usually given subcutaneously in patients with diabetes mellitus type 1 to treat severe hypoglycemia.

- Glucagon has also been used in dual-hormone artificial pancreas with some improvement in glucose control.

What are the new findings?

- Glucagon injected intraperitoneally gives a higher glucose response 4 min after administration and affects blood glucose for a shorter period compared with subcutaneous injection.

How might these results change the focus of research or clinical practice?

- The present results should encourage research on the feasibility of combined intraperitoneal administration of insulin and glucagon as part of an artificial pancreas in humans.

is important, as chronic hyperglycemia may induce neuropathy, nephropathy, retinopathy, and cardiovascular diseases. ${ }^{12}$ Achieving euglycemia is challenging due to slow absorption and delayed glucose-lowering effect of subcutaneously administrated insulin. This makes it difficult to achieve optimal postprandial glucose control without the risk of subsequent hypoglycemia. ${ }^{3}$ Repeated and frequent episodes of hypoglycemia are associated with impaired neuroendocrine counter-regulation and symptom perception and deterioration of cerebral functions and may lead to hypoglycemia unawareness. ${ }^{4}$ Therefore, the central nervous system's adaptation to frequent shortterm hypoglycemias may contribute to the increased incidence of severe hypoglycemia. ${ }^{5}$ Despite many small improvements in the treatment of DM1 during the last decades, 
hypoglycemia remains a challenge for many patients with DM1. ${ }^{6-8}$

Glucagon is used for treating severe hypoglycemia when patients with DM1 are unconscious and unable to consume carbohydrates. The standard treatment for adults is an intramuscular, intravenous or subcutaneous injection of $1 \mathrm{mg}$ of glucagon. Whether this dose is optimal for all routes of administration has hardly been studied. ${ }^{910}$ The glucose increasing effect depends on the dose of the injected glucagon, ${ }^{11}$ amount of liver glycogen ${ }^{12}$ and baseline blood glucose level. ${ }^{13}$ When studied in healthy men, there seems to be no major difference in the glucose effect between intramuscular and subcutaneous administration. ${ }^{14}{ }^{15}$ Recently, smaller glucagon doses have been used with success to avoid mild or impending hypoglycemia in children and adults. ${ }^{6} 1116$ Glucagon used as an emergency nasal spray and nasal powder has been launched as an alternative route of administration, providing a success rate in treating hypoglycemia similar to intramuscular injections. ${ }^{17-19}$

Recent work on algorithm-steered insulin delivery (ie, artificial pancreas (AP)) provides improvements to glucose regulation. Unfortunately, this automatically controlled (closed loop) delivery of insulin carries certain limitations, as nearly all recent developments depend on a double subcutaneous approach, that is, both glucose measurements and insulin delivery are in the subcutaneous tissue. The limitations are due to slow subcutaneous glucose dynamics secondary to both delayed and slow subcutaneous insulin absorption, which unavoidably lead to alternating periods of either a lack or excess of circulating insulin. ${ }^{20}$ To solve the challenge of relative insulin excess, some research groups have incorporated glucagon as a counter-regulator in the AP system, to counteract imminent hypoglycemia, that is, a dual hormonal $\mathrm{AP}^{72122}$ Despite achieving as low as 3\% of time in hypoglycemic range during day and night closed loop control, hypoglycemia still remains a substantial daytime problem also in this subcutaneous dual-hormone approach. ${ }^{23}$ Therefore, new routes should be explored to find better solutions for prevention of hypoglycemia.

Intraperitoneal glucagon administration has only been reported from a few animal studies. ${ }^{24} 25$

The main aim of this study was to compare the glucose increasing effect after subcutaneous and intraperitoneal delivery of glucagon, and to investigate the potential for intraperitoneal delivery of small doses of glucagon in an AP. Intravenous delivery of hormones is less realistic in free-living conditions. Therefore, intravenous route was only included in the study as an additional route to obtain more information on glucose dynamics after glucagon delivery, and not included as a main outcome in the paper. We hypothesized that the glucose response is faster after intraperitoneal compared with subcutaneous administration of glucagon. To investigate this hypothesis, we compared the immediate glucose response after intraperitoneal, subcutaneous and intravenous administration of glucagon in an animal model.

\section{RESEARCH DESIGN AND METHODS}

\section{Pilot study}

A pilot study was performed on 10 rats to refine the experimental protocol and to determine the glucagon dose to be used in the main study. Detailed explanation is available in the online supplementary material.

\section{Animals}

In the main study male Sprague Dawley rats $(n=20)$ (initial weight 470-615 g; Janvier Labs, France), in groups of three, were kept in plastic solid bottom cages $(515 \times 381 \times 256$ $\mathrm{mm}$, Tecniplast, Italy) on sawdust. The rats were acclimatized to the animal facility and maintained on 12-hour light-12-hour dark photoperiod at $20-24^{\circ} \mathrm{C}$ and a relative humidity of $55 \% \pm 5 \%$. They were fed expanded pellets (Special Diets Services RM1 for rats, UK) and fresh water was available ad libitum. To reduce stress and the possible effect of stress on glucose levels, the rats were trained to accept general handling and use of a restrainer (Harvard Apparatus, Holliston, USA) for 3 weeks prior to the start of experiments.

\section{Intervention groups and randomization}

The assignment to intervention groups $(n=20)$ and the order of procedures in each rat were randomized by creating random permutations of treatment and intervention groups. The glucagon dose was $5 \mu \mathrm{g} / \mathrm{kg}$ body weight (BW) for all interventions except placebo. All rats $(n=20)$ received intraperitoneal and subcutaneous injection of glucagon, 15 of the rats received placebo intraperitoneal injections of $1 \mathrm{~mL} / \mathrm{kg} \mathrm{BW}$ of isotonic saline. The volume of placebo injection $(1 \mathrm{~mL} / \mathrm{kg} \mathrm{BW})$ was similar to the intraperitoneal glucagon injection (approximately $500 \mu \mathrm{L}$ ). To obtain information also after intravenous delivery of glucagon, five of the rats were administered intravenous glucagon (see online supplementary tables $2 \mathrm{a}$ and $\mathrm{b}$ ). There was at least 1 week between each test procedure on each rat. To avoid bias based on metabolic individualities, trials were performed in the 12-hour light period, and all procedures in each individual rat were done at approximately the same time as of the light cycle. Group size was determined by the resource equation method. ${ }^{26}$

\section{Technical challenges}

The rats were monitored for the entire sampling period (70 min) and surveyed for signs of stress. Except when the rats were anesthetized, they were kept in restrainers to facilitate blood sampling. Restrainers of two different sizes were tested before the start of the experiment. For most of the rats, the restrainers were either too large or too small. Thus, the larger restrainer was used for all rats, and a paper tissue was rolled up and taped vertically to the inside of the restrainer at a level behind the rat's shoulder, to prevent the smaller rats from turning around inside the restrainer.

\section{Procedures}

Food was removed 1 hour before the start of the procedure and water was available ad libitum. The individual 
glucagon and octreotide doses were based on the animal's weight on the day of the procedure.

\section{Endogenous glucagon secretion}

To suppress the endogenous glucagon and insulin secretion during the procedures, all rats received two subcutaneous injections of $10 \mu \mathrm{g} / \mathrm{kg}$ BW octreotide (Sandostatin $200 \mu \mathrm{g} / \mathrm{mL}$, Novartis Europharm, UK). The first injection was given approximately $30 \mathrm{~min}$ before the start of each procedure and the second at the time of glucagon/ placebo injection. Octreotide was given subcutaneously in the neck, but not at the same location as the subcutaneous glucagon injection.

\section{Anesthesia}

To prevent accidental movements in the time of the procedure, the rats were anesthetized with isoflurane (Isoflurane, Baxter, Oslo, Norway; $5 \%$ IF, 95\% air in chamber; $2 \%$ IF, $95 \%$ air on face mask) for two short intervals at the start of each procedure. During the first anesthesia period, a cut in the tail for collection of blood samples was made. During the second anesthesia period, an injection of glucagon or placebo was given. When required, additional anesthesia was provided to rats showing signs of stress while kept in the restrainer.

\section{Glucagon challenge}

Glucagon (Glucagon, Novo Nordisk, Denmark) was diluted by $0.9 \% \mathrm{NaCl}$ to a concentration of $5 \mu \mathrm{g} / \mathrm{mL}$ and the rats were given $5 \mu \mathrm{g} / \mathrm{kg}$ BW. Glucagon solutions were kept in a refrigerator and used the same day they were made. Solutions were warmed to approximately body temperature just before administration. Subcutaneous glucagon was injected at the back of the neck, and intraperitoneal glucagon and placebo (an equal volume of $0.9 \% \mathrm{NaCl}$ ) in the lower part of the abdomen, with the rat held at an angle after its hind legs. Intravenous glucagon was given in the lateral tail vein that was not currently used for blood sampling.

\section{Glucose measurement}

After disinfecting the skin, a 6-9 $\mathrm{mm}$ cut was made with a straight-edged scalpel over the lateral tail vein two-thirds down the length of the tail for blood sampling. Samples were collected 10, 5 and 1 min prior to glucagon injection, and 2, 4, 6, 8, 10, 15, 20, 25, 30, 40, 50, and $60 \mathrm{~min}$ after the glucagon or placebo injections. Whenever needed, the vein was carefully reopened with the tip of the scalpel to ensure sufficient blood flow for sampling.

Blood for glucose analyses was collected directly in heparinized capillary tubes ( $35 \mu \mathrm{L}$, Clinitubes, Radiometer Medical ApS, Brønshøj, Denmark), and stored on ice for a maximum of $30 \mathrm{~min}$ before analysis on a blood gas analyzer (Radiometer ABL 725, Radiometer Medical ApS). To ensure sufficient blood flow for sampling, the vein was gently stroked from the base of the tail and toward the wound, and the first small drop of blood was removed. For the third intervention, both veins had been used for sampling at former trials, and the new cut was made proximal to the older cut. Occlusion of the rat's tail vein occurred in only one rat, and in this case the vein on the other side of the tail was used.

\section{Animal welfare}

The rats were given non-steroidalanti-inflammatory drugs (Metacam vet, Boehringer Ingelheim Vetmedica) $1 \mathrm{mg} / \mathrm{kg} \mathrm{BW}$ as a single subcutaneous injection at the end of the two first procedures. A suture, to close the wound and stop the bleeding at the end of the procedure, was necessary in 19 cases. The wounds healed well after sampling regardless of the wound being sutured or not, and no wound infections were observed. After the third procedure, the rats were euthanized with an intravenous injection of pentobarbital $(100 \mathrm{mg} / \mathrm{kg}$ ) (Norges Apotekerforening, Norway) under isoflurane anesthesia.

\section{Statistical analysis}

The relationship between glucose levels and time was analyzed for all interventions using a mixed linear model with the combination of time and treatment as the fixed effect. The dependent variable was defined as log glucose concentration to achieve normal distribution. To account for multiple measurement series on each rat, rat identification was included as a random effect. To account for dependence within each series, the error term for each series was specified as a first-order autoregressive process AR (1) series accounting for minutes between measurements. Mean changes in glucose concentrations from -1 min to 2-60 min for the four treatments were compared using the Wald test. Maximum concentration and time until maximum concentration of the estimated model for the treatments were compared using the Mann-Whitney $\mathrm{U}$ test. To eliminate the effect of placebo intervention on the glucose response, the mean value of the 15 placebo interventions was subtracted from the mean value of the 20 subcutaneous and intraperitoneal interventions and the mean value of the 5 intravenous interventions at the given time points. All interventions are compared as models; therefore, comparison between unequal groups is allowed. ${ }^{27}$ The software package $\mathrm{R}$ was used to analyze the data. ${ }^{28}$ All values in the text are given as mean \pm SE of the mean, unless stated otherwise. Differences between the group means were considered statistically significant at a threshold of $\mathrm{p} \leq 0.05$.

\section{RESULTS}

In general, the rats stayed calm during the experimental procedures. Thirteen incidents occurred during 60 procedures, in which the rats turned around inside the restrainers or showed signs of stress and consequently were taken out of the restrainer and repositioned. A similar number of incidences were found in all interventions (four during the intraperitoneal, four during the subcutaneous and three during the intravenous intervention). These incidents included two rats in whom stress was observed during three procedures (intraperitoneal, subcutaneous and intravenous). Two incidents of stress 
were observed during the placebo procedure; however, no increase in blood glucose levels from baseline was observed (data not shown).

Additional anesthesia during blood sampling was needed during 10 intraperitoneal, 10 subcutaneous, 5 intravenous, and 5 placebo interventions, and the mean \pm SD time in anesthesia was $13.8 \pm 5.5 \mathrm{~min}$ $(14.05 \pm 5.47,13.55 \pm 4.37,20 \pm 9.43$ and $12 \pm 4.85$, respectively). The rats were conscious for the rest of the 70 min procedure. After individually analyzing data from the 16 rats which received the longest duration of anesthesia (time in anesthesia 15-31 min, subcutaneous $n=5$, intraperitoneal $n=5$, placebo $n=3$ and intravenous $n=3$ ), only two rats (subcutaneous intervention, $n=2$ ) showed prolonged elevated glucose levels and no decrease of glucose values at the end of the intervention (at $60 \mathrm{~min}$ ) (data not shown).

\section{Glucose level}

For calculation of glycemic state for rats at the beginning of the interventions, a mean baseline glucose was calculated according to the mean of three measurements preceding the intervention $(-10,-5$ and $-1 \mathrm{~min})$, and in addition a mean $\pm \mathrm{SD}$ in each intervention group was calculated. Blood glucose levels at the beginning of the intraperitoneal, subcutaneous, intravenous and placebo interventions were $6.72 \pm 0.90,6.47 \pm 0.81,6.17 \pm 1.12$ and $6.51 \pm 0.81 \mathrm{mmol} / \mathrm{L}$, respectively (see online supplementary figure 1).
Compared with placebo, glucose was significantly increased $4 \mathrm{~min}$ after intraperitoneal glucagon injection $(\mathrm{p}=0.009, \mathrm{n}=20)$, and $8 \mathrm{~min}$ after subcutaneous $(\mathrm{p}=0.002, \mathrm{n}=20)$ and intravenous $(\mathrm{p}<0.001, \mathrm{n}=5)$ injections (figure 1).

Comparing intraperitoneal glucagon injections with subcutaneous, the glucose increase after intraperitoneal glucagon was significantly higher at $4 \mathrm{~min}(\mathrm{p}=0.019)$ and significantly lower at $40(\mathrm{p}=0.005)$ and $50 \mathrm{~min}$ $(\mathrm{p}=0.011)$ (figure 1). Comparing intravenous glucagon injections with intraperitoneal, the glucose increase after intravenous injection was significantly higher at $20 \mathrm{~min}$ $(p=0.001)$. At the other time points, no significant differences were observed.

An increase in glucose levels was seen after all three routes of glucagon delivery, but there was no significant difference $(\mathrm{p}=0.52)$ in absolute maximum blood glucose value after intraperitoneal glucagon injection (9.74 mmol/L) compared with subcutaneous injection $(10.3 \mathrm{mmol} / \mathrm{L})$. The estimated time until the maximum glucose value was significantly shorter $(\mathrm{p}=0.003)$ after intraperitoneal glucagon injection $(25 \mathrm{~min})$ versus subcutaneous glucagon injection (35 min) (see online supplementary figure 1).

\section{DISCUSSION}

The results of this study indicate that the glucose response in rats comes earlier when glucagon is injected

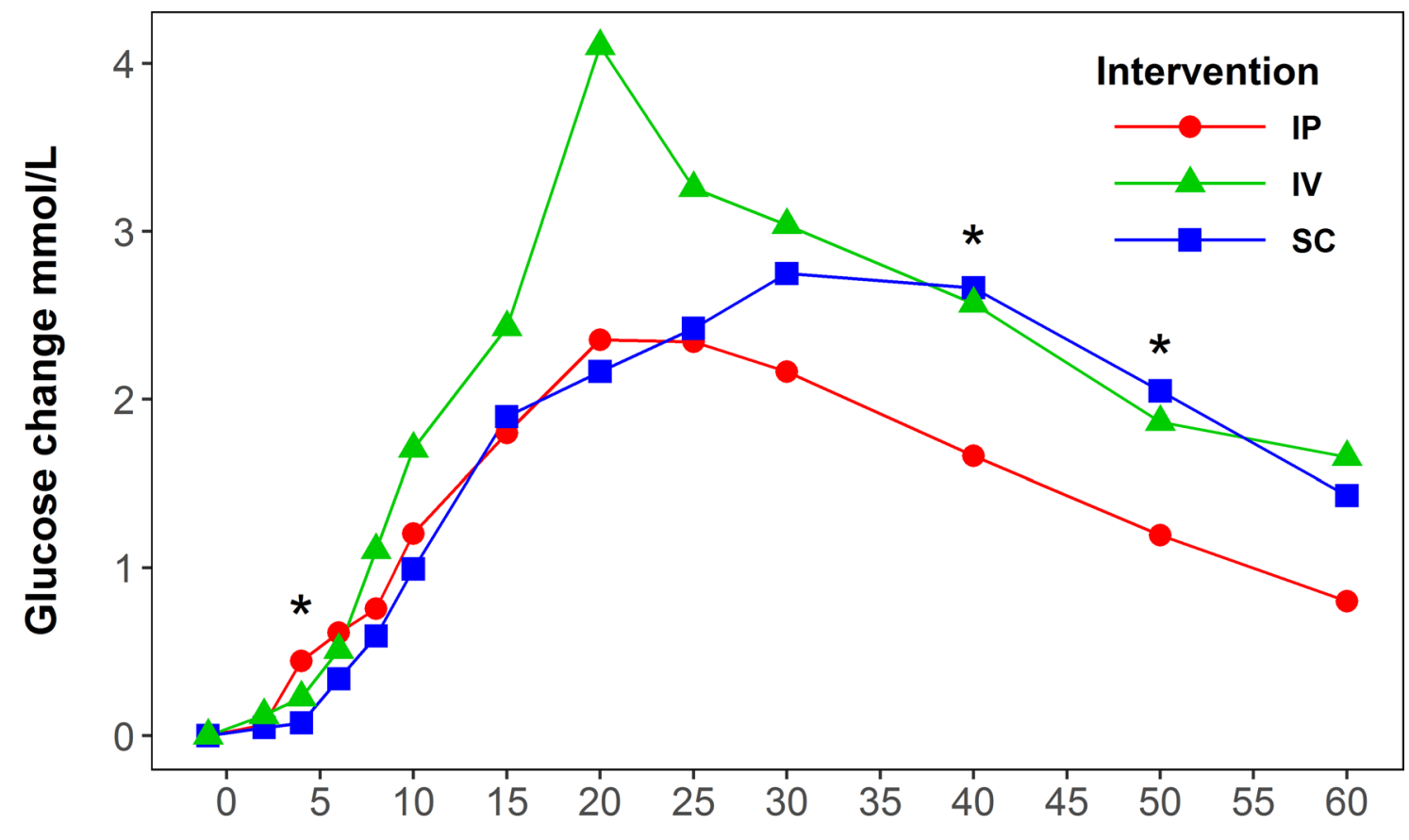

\section{Time after glucagon injection (minutes)}

Figure 1 Estimated glucose delta values (mmol/L) after glucagon injection $(5 \mu \mathrm{g} / \mathrm{kg})$ in octreotide-treated rats. Green line represents intravenous (IV) intervention, blue line represents subcutaneous (SC) intervention, and red line represents intraperitoneal (IP) intervention. The glucose response for the placebo group has been subtracted from all groups presented in the figure. *Represents significant difference between intraperitoneal and subcutaneous of glucagon delivery. Note: For one rat in intraperitoneal intervention, delta values were calculated using the -5 minute measurement ( -1 minute for other rats). 
intraperitoneally than when injected subcutaneously. Second, the maximum effect of glucose increase appears earlier, and the glucose response diminishes faster after intraperitoneal compared with subcutaneous glucagon injection.

The peritoneal lining is highly vascularized, and the blood capillary density in the peritoneal lining varies between individuals (higher amount in infants (0-1 year) and adults, and lower amount in children).${ }^{29}$ There is no systematic variation in histological parameters in different parts of the peritoneum. ${ }^{29} 30$ Compared with subcutaneous absorption, peritoneal absorption may be faster due to a shorter distance to reach the capillaries and easier diffusion into the bloodstream. From animal studies, we know that most of the intraperitoneally injected insulin enters the portal vein and passes the liver before entering the systemic circulation. ${ }^{31-33}$ Consequently, after intraperitoneal delivery, glucagon probably reaches the liver both earlier and at a higher concentration and thereby promotes hepatic gluconeogenesis earlier as compared with subcutaneously injected glucagon. This is compatible with our observation of faster glucose increase after intraperitoneal injection of glucagon compared with other injection routes. It also fits well with the observed earlier maximum glucose response after intraperitoneally injected compared with subcutaneously injected glucagon. Interestingly, time until maximum blood glucose increase after subcutaneous injection in our animal model is similar to what is observed in humans with diabetes. ${ }^{11}$

Our finding of an earlier rise in blood glucose after intraperitoneally injected glucagon, compared with subcutaneous injection, is difficult to compare with previous studies as blood glucose was measured at different time points and intervals. In previous studies, blood glucose was only measured $20^{24}$ and $30^{25}$ min after glucagon injection. Moreover, the study by Zlotnik $e t a l^{25}$ provided only data of intraperitoneal glucagon injection.

In the present study, blood glucose was lower after intraperitoneal compared with subcutaneous injection of glucagon at time points 40 and 50 min. Fifty minutes after glucagon injection, a declining blood glucose was observed in all routes (intraperitoneal, subcutaneous and intravenous) of administration. This differs from a previous study in rats, ${ }^{25}$ where after intraperitoneal injections of glucagon a significant rise in glucose levels was observed after 30, 60, 90 and $120 \mathrm{~min}$. However, after 60-90 min, a flattening of the blood glucose curve was observed and at $120 \mathrm{~min}$ blood glucose subsequently decreased. This discrepancy between the previous and the present results may depend on the fact that in the previous study, rats were anesthetized during the whole procedure with isoflurane,${ }^{25}$ while in the present study we limited isoflurane use as much as possible (see online supplementary material). We also treated the rats with octreotide to inhibit endogenous release of insulin and glucagon during the experiments. Interestingly, in the previous study no difference in plasma glucose was observed in the control group, while in our pilot study, with extended use of isoflurane, we observed an increase in glucose levels (see online supplementary material). A glucose increasing effect of isoflurane has been described previously. ${ }^{34}$

Loxham et $a l^{24}$ demonstrated results similar to our study, where, after intraperitoneal injection of glucagon in non-diabetic rats, the glucose response after intraperitoneal administration was higher after $20 \mathrm{~min}$ and lower after 45 min compared with subcutaneous administration. Baseline glucose levels were also similar to ours. However, the authors did not mention whether anesthetics were used. ${ }^{24}$ Noteworthy, Loxham $e t a l^{24}$ suggested that different strains of rats may react differently to a sudden rise in counter-regulators (in this case glucagon), making comparison between different strains of rats difficult.

Our glucagon dose of $5 \mu \mathrm{g} / \mathrm{kg} \mathrm{BW}$ was only $2.5 \%$ of the dose used by Loxham et $a l^{24}(200 \mu \mathrm{g} / \mathrm{kg})$ and only $1 \%$ of the dose used by Zlotnik et $a l^{25}(50 \mu \mathrm{g} / 100 \mathrm{~g})$. We do not have information about why these particular doses were chosen. Another aspect is that previous authors used naïve rats, whereas the rats in the present study were treated with octreotide. ${ }^{35}$ Our study provides information about possible doses of glucagon with which glucagon saturation is reached (see online supplementary material). It seems that in our study a glucagon dose of $5 \mu \mathrm{g} /$ $\mathrm{kg}$ BW was appropriate (see online supplementary material) based on the observed blood glucose increase of around $3 \mathrm{mmol} / \mathrm{L}$ in all injection routes.

Our study is not the first to explore the effect of smaller doses of glucagon. The effect of smaller doses than the standard $1 \mathrm{mg}$ of glucagon (commonly used in cases of serious hypoglycemia) has recently been examined in humans. ${ }^{611}$ Glucagon may induce nausea and vomiting, and these side effects may be related to the size of the injected dose and the subsequent higher levels of glucagon in the systemic circulation. In patients with DM1 there seems to be a dose-response relationship between subcutaneous glucagon doses ranging from 0.11 $\mathrm{mg}$ to $1.0 \mathrm{mg}$ and the glucose response. ${ }^{11}$ Mini-doses of glucagon are effective in treating mild to moderate hypoglycemic episodes in both children ${ }^{636}$ as well as adults. ${ }^{16}$

The motivation for performing this study was to investigate and compare different administration routes of glucagon and explore if glucagon administration intraperitoneally would provide some benefits compared with subcutaneous injection, aspects of importance for the development of a dual hormonal AP. Ideally, a dual-hormone AP should prevent hypoglycemias with small and, if necessary, repeated doses of glucagon. ${ }^{737}$ Minimizing the amount of exogenous glucagon needed to counteract hypoglycemia is important to avoid the depletion of liver glycogen, to reduce the side effects, such as nausea and vomiting, and to avoid reactive hyperglycemia. ${ }^{11}$

A small dip in glucose values was observed prior to the injection of glucagon or saline, that is, during all procedures (see online supplementary figure 1 ). The reason 
for this is unclear, but the equal response during all procedures is an indication of consistency of the experimental protocol for all the procedures during the first part of the experiment and thereby a sign of quality of the present study.

\section{Strengths and limitations of the study}

The following are the strengths of the present study: (1) placebo-treated animals - the importance is illustrated by the fact that, although not significantly, the glucose levels tended to fluctuate also during the placebo procedures; (2) limited use of anesthesia, which may have major impact on glucose homeostasis; (3) systematic training of the rats to the procedures for weeks ahead of the test procedures (both pilot rats and trial rats) to minimize the stress response during the test procedures; and (4) randomization of the order of treatment in each rat.

The following are among the limitations of the present study: (1) With unguided injections into the abdominal cavity, we cannot be sure that all the glucagon or placebo was administrated in the peritoneal space. However, glucagon and placebo saline were injected by the same procedure in anesthetized rats (injection could be done without experiencing unexpected movements of rat); therefore, possible deviation from intraperitoneal delivery should be equal between groups. (2) A few rats were stressed during the procedures, which might affect the blood glucose levels. However, in rats receiving placebo intervention, under signs of stress, the blood glucose level did not increase significantly (see online supplementary material). (3) Additional anesthesia was needed for some rats in all interventions; however, as it was described in the results, prolonged increased glucose levels were observed only in 2 out of the 16 rats which were exposed to the longest duration of anesthesia in the main study. Therefore glucose level increase at the end of the intervention can be individual response, not anesthesia-induced. (4) Rats were fasted differently (between 1 and 3 hours) depending on the order of performing the procedure (intraperitoneal, subcutaneous, intravenous or placebo). However, to avoid bias, all experiments on the same rat were conducted at approximately the same time as of the light cycle.

\section{CONCLUSION}

Blood glucose increased faster when glucagon was injected in the peritoneal cavity compared with subcutaneous glucagon delivery in octreotide-treated rats. The maximum glucose response was reached earlier and the decline in glucose response was also faster. If repeatable in humans, a more rapid glucose response may be of importance in a dual-hormone AP using the intraperitoneal route for administration of insulin and glucagon.

\section{Author affiliations}

${ }^{1}$ Department of Clinical and Molecular Medicine, Faculty of Medicine and Health Sciences, NTNU - Norwegian University of Science and Technology, Trondheim, Norway
${ }^{2}$ Department of Endocrinology, St Olavs Hospital, Trondheim, Norway ${ }^{3}$ Department of Engineering Cybernetics, Faculty of Information Technology and Electrical Engineering, NTNU - Norwegian University of Science and Technology, Trondheim, Norway

Acknowledgements The animal experiments were conducted at the Comparative Medicine Core Facility (CoMed), Norwegian University of Science and Technology (NTNU). CoMed is funded by the Faculty of Medicine and Health Sciences at NTNU and the Central Norway Regional Health Authority. We thank Associate Professor Øyvind Salvesen, Faculty of Medicine and Health Sciences, NTNU, for help with the statistical analysis, and Gita Nagarajah for her work on the glucose analysis.

Contributors ID-F and MKÅ completed the trial, collected and analyzed the data, wrote and edited the manuscript, and are the guarantors of the work. ALF, SMC and SCC contributed to the discussion, and reviewed and edited the manuscript. All authors contributed to the development of the protocol.

Funding The Norwegian Research Council (NRC) is funding the Double Intraperitoneal Artificial Pancreas project (project number 248872) as part of the Centre for Digital Life Norway (digitallifenorway.org). The study is also supported by a scholarship from the Central Norway Regional Health Authority (grant nr 2014/23166) and the Norwegian Medical Association Johan Selmer Kvanes Endowment. The funding sources had no role in the collection, analysis or interpretation of the data.

Competing interests None declared.

Patient consent Not required.

Ethics approval The study was approved by the Norwegian Food Safety Authority (FOTS-ID 10922) and was in accordance with 'The Norwegian Regulation on Animal Experimentation' and 'Directive 2010/63/EU on the protection of animals used for scientific purposes'.

Provenance and peer review Not commissioned; externally peer reviewed. Data sharing statement Additional online data supplement is available.

Open access This is an open access article distributed in accordance with the Creative Commons Attribution Non Commercial (CC BY-NC 4.0) license, which permits others to distribute, remix, adapt, build upon this work non-commercially, and license their derivative works on different terms, provided the original work is properly cited, appropriate credit is given, any changes made indicated, and the use is non-commercial. See: http://creativecommons.org/licenses/by-nc/4.0/

\section{REFERENCES}

1. Shaikh M, Shaikh B, Shah NA. Comparative study on lipid changes in glycemic uncontrolled diabetes type 1 and type 2. J Liaquat Uni Med Health Sci 2009;8:201-4.

2. Epidemiology of severe hypoglycemia in the diabetes control and complications trial. The DCCT Research Group. Am J Med 1991;90:450-9.

3. Guerci B, Sauvanet JP. Subcutaneous insulin: pharmacokinetic variability and glycemic variability. Diabetes Metab 2005;31:4S7-24.

4. Lingenfelser T, Overkamp D, Renn W, et al. Insulin-associated modulation of neuroendocrine counterregulation, hypoglycemia perception, and cerebral function in insulin-dependent diabetes mellitus: evidence for an intrinsic effect of insulin on the central nervous system. J Clin Endocrinol Metab 1996;81:1197-205.

5. Lingenfelser T, Renn W, Sommerwerck U, et al. Compromised hormonal counterregulation, symptom awareness, and neurophysiological function after recurrent short-term episodes of insulin-induced hypoglycemia in IDDM patients. Diabetes 1993;42:610-8.

6. Hartley M, Thomsett MJ, Cotterill AM. Mini-dose glucagon rescue for mild hypoglycaemia in children with type 1 diabetes: the Brisbane experience. J Paediatr Child Health 2006;42:108-11.

7. Haidar A, Rabasa-Lhoret R, Legault L, et al. Single- and dualhormone artificial pancreas for overnight glucose control in type 1 diabetes. J Clin Endocrinol Metab 2016;101:214-23.

8. Jacobs PG, El Youssef J, Reddy R, et al. Randomized trial of a dualhormone artificial pancreas with dosing adjustment during exercise compared with no adjustment and sensor-augmented pump therapy. Diabetes Obes Metab 2016;18:1110-9.

9. White M, Zacharin MR, Werther GA, et al. Intravenous glucagon in a deliberate insulin overdose in an adolescent with type 1 diabetes mellitus. Pediatr Diabetes 2016;17:66-9.

10. Blauw $H$, van Bon AC, Koops $R$, et al. Performance and safety of an integrated bihormonal artificial pancreas for fully automated glucose control at home. Diabetes Obes Metab 2016;18:671-7. 
11. Blauw H, Wendl I, DeVries JH, et al. Pharmacokinetics and pharmacodynamics of various glucagon dosages at different blood glucose levels. Diabetes Obes Metab 2016;18:34-9.

12. Castle JR, Engle JM, El Youssef J, et al. Factors influencing the effectiveness of glucagon for preventing hypoglycemia. J Diabetes Sci Technol 2010;4:1305-10.

13. Rivera N, Ramnanan CJ, An Z, et al. Insulin-induced hypoglycemia increases hepatic sensitivity to glucagon in dogs. J Clin Invest 2010;120:4425-35.

14. Graf CJ, Woodworth JR, Seger ME, et al. Pharmacokinetic and glucodynamic comparisons of recombinant and animal-source glucagon after IV, IM, and SC injection in healthy volunteers. J Pharm Sci 1999;88:991-5.

15. Mühlhauser I, Koch J, Berger M. Pharmacokinetics and bioavailability of injected glucagon: differences between intramuscular, subcutaneous, and intravenous administration. Diabetes Care 1985;8:39-42.

16. Haymond MW, DuBose SN, Rickels MR, et al. Efficacy and safety of mini-dose glucagon for treatment of nonsevere hypoglycemia in adults with type 1 diabetes. J Clin Endocrinol Metab 2017;102:2994-3001.

17. Boido A, Ceriani V, Pontiroli AE. Glucagon for hypoglycemic episodes in insulin-treated diabetic patients: a systematic review and meta-analysis with a comparison of glucagon with dextrose and of different glucagon formulations. Acta Diabetol 2015;52:405-12.

18. Rickels MR, Ruedy KJ, Foster NC, et al. Intranasal glucagon for treatment of insulin-induced hypoglycemia in adults with type 1 diabetes: a randomized crossover noninferiority study. Diabetes Care 2016;39:264-70.

19. Sherr JL, Ruedy KJ, Foster NC, et al. Glucagon nasal powder: a promising alternative to intramuscular glucagon in youth with type 1 diabetes. Diabetes Care 2016;39:555-62.

20. Christiansen SC, Fougner AL, Stavdahl $\varnothing$, et al. A review of the current challenges associated with the development of an artificial pancreas by a double subcutaneous approach. Diabetes Ther 2017;8:489-506.

21. Taleb N, Emami A, Suppere C, et al. Efficacy of single-hormone and dual-hormone artificial pancreas during continuous and interval exercise in adult patients with type 1 diabetes: randomised controlled crossover trial. Diabetologia 2016;59:2561-71.

22. Haidar A, Messier V, Legault L, et al. Outpatient 60-hour day-andnight glucose control with dual-hormone artificial pancreas, singlehormone artificial pancreas, or sensor-augmented pump therapy in adults with type 1 diabetes: an open-label, randomised, crossover, controlled trial. Diabetes Obes Metab 2017;19:713-20.

23. Russell SJ, El-Khatib FH, Sinha M, et al. Outpatient glycemic control with a bionic pancreas in type 1 diabetes. $N$ Engl J Med 2014;371:313-25.

24. Loxham SJ, Teague J, Poucher SM, et al. Glucagon challenge in the rat: a robust method for the in vivo assessment of Glycogen phosphorlyase inhibitor efficacy. J Pharmacol Toxicol Methods 2007;55:71-7.

25. Zlotnik A, Gruenbaum BF, Klin Y, et al. The effects of insulin, glucagon, glutamate, and glucose infusion on blood glutamate and plasma glucose levels in naive rats. J Neurosurg Anesthesiol 2011;23:323-8.

26. Charan J, Kantharia ND. How to calculate sample size in animal studies? J Pharmacol Pharmacother 2013;4:303-6.

27. Engle RF. wald, likelihood ratio, and lagrange multiplier tests in econometrics. In: . Handbook of econometrics. Elsevier, 1984: 775-826.

28. R: a language and environment for statistical computing. Vienna, Austria: R Foundation for Statistical Computing, 2017.

29. Schaefer B, Bartosova M, Macher-Goeppinger S, et al. quantitative histomorphometry of the healthy peritoneum. Sci Rep 2016;6:21344.

30. Slater NJ, Raftery AT, Cope GH. The ultrastructure of human abdominal mesothelium. J Anat 1989;167:47-56.

31. Fischer U, Freyse EJ, Salzsieder E, et al. Artificial connection between glucose sensing and insulin delivery: implications of peritoneal administration. Artif Organs 1992;16:151-62.

32. Fritze K, Fischer U, Freyse EJ, et al. Intraindividual comparison of pharmacokinetics of insulin after intravenous, portal, subcutaneous and peritoneal administration. Exp Clin Endocrinol 1988;92:297-306.

33. Schade DS, Eaton RP, Davis T, et al. The kinetics of peritoneal insulin absorption. Metabolism 1981;30:149-55

34. Wren-Dail MA, Dauchy RT, Blask DE, et al. Effect of isoflurane anesthesia on circadian metabolism and physiology in rats. Comp Med 2017;67:138-46.

35. Schmid HA, Brueggen J. Effects of somatostatin analogs on glucose homeostasis in rats. J Endocrinol 2012;212:49-60.

36. Hasan KS, Kabbani M. Mini-dose glucagon is effective at diabetes camp. J Pediatr 2004;144:834.

37. Castle JR, Engle JM, El Youssef J, et al. Novel use of glucagon in a closed-loop system for prevention of hypoglycemia in type 1 diabetes. Diabetes Care 2010;33:1282-7. 